\title{
Dysphagia: Approach to Assessment and Treatment
}

\author{
Lalsa SP* \\ Clinical Audiologist and Speech Language Pathologist, Hearing First University, Canada
}

Submission: April 22, 2017; Published: June 28, 2017

*Corresponding author: Lalsa Shilpa Perepa, Clinical Audiologist and Speech Language Pathologist, Hearing First University, Canada, Tel: +16478655783; Email: lalsashilpa.311@gmail.com

\section{Introduction}

Dysphagia is a medical term used to describe a swallowing disorder. It may refer to a swallowing disorder involving any one of the 3 stages of swallowing: oral, pharyngeal, esophageal. It is not a primary medical diagnosis, but a symptom of a disease, \& therefore is described most often by its clinical characteristics. Dysphagia is delay in or misdirection of a fluid or solid bolus as it moves from mouth to the stomach. Delay in or misdirection of the food bolus may interfere with functional oral intake.

\section{The nature of dysphagia}

Aspiration occurs whenever food enters the airway below the true vocal folds. Aspiration can occur before, during, or after the swallow.

\section{Aspiration before the swallow}

Aspiration occurs before the swallow in the case of a delayed or absent swallow initiation. It may also be the result of poor tongue control, which allows food to trickle into the pharynx while the patient is still chewing. Apparently, a "neurological override" exists which prevents the initiation of the swallow while one is still chewing [1].

\section{Aspiration during the swallow}

Aspiration occurs during the swallow when the vocal folds fail to adduct or the larynx fails to elevate. (Remember that this type of dysphagia is uncommon. Only $5 \%$ of dysphagias involve problems with airway closure).

\section{Aspiration after the swallow}

Aspiration can occur after the swallow in several different circumstances: The patient may pocket food in the oral cavity. Later, when he or she lies down to sleep, the food will fall down into the airway. Food may get stuck in the pharyngeal recesses. This happens to everyone, but someone with a normal system would realize that the food was there and swallow again. A CVA or TBI patient may have a sensory impairment and allow the food to drop into the larynx. Due to reduced laryngeal elevation, food may remain on top of the larynx (Logemann, 1989).

\section{Signs \& symptoms of dysphagia}

Early identification and treatment (Tx) may help avoid adverse medical complications such as under nutrition or respiratory infection. Because a variety of medical specialists can be involved in the care of the patient with dysphagia, all must be capable of detecting the signs \& symptoms characteristics of dysphagia. Some symptoms may be overt, such as those in the patient who coughs while eating, where as others may not be overt, such as those in the patient who may not have a swallowing complaint but comes to the swallowing specialist with a history of unexplained pneumonia.

A radiographic evaluation of swallowing may reveal that food or fluid is silently entering the air way during swallow, resulting in aspiration.

Symptoms of dysphagia: symptoms are usually are defined as any perceptible change in bodily function that the patient notices. This change eventually leads the patient to seek medical help when it causes pain or discomfort or negatively impacts his/her life style. Some people have adverse medical symptoms \& ignore them until the severity of their problem significantly affects their physiologic or mental health. Others seek immediate medical attention. Both groups may be diagnosed with a disorder that is similar in type \& severity.

Patient description: the physical examination of a patient with dysphagia may begin by asking him or her to describe the symptoms. Because dysphagia often is secondary to neurological disease that also may compromise communication skills, not all patients can provide a report of their symptoms.

Because of cortical deficits, others may give unreliable or scant information. They make changes in their eating habits to accommodate their symptoms, such as chewing food more finely or eliminating troublesome items from their menu. Others know that they are having difficulty swallowing but have a difficult time describing the specifics of their symptoms. Often it is difficult for them to remember how long those symptoms have been apparent. 
This may be due to the inherent flexibility of the swallowing tract to accommodate changes in function. For patients who are able to communicate symptoms of their dysphagia, a detailed description may be useful in helping establish a diagnosis. Detailed descriptions also may be used to help the examiner focus on the types of diagnostic tests that may be most useful in delineating the source of the patient's complaint. Some clinicians find it useful to explore a patient's dysphagic symptoms by questionnaire. This method may help ensure that all relevant questions relating to the patient's symptoms are addressed by the examiner. It also gives the patient a chance to think carefully about his/her symptoms before responding.

Obstruction: one of the common complaints from dysphagic patients is that food or fluids "gets stuck". Most often, they report that the sticking sensation is in the throat or esophagus. Some patients do not use the word stuck but may use the word "fullness". When they localize the feeling of obstruction to the throat, they often describe their complaints as "a lump in the throat" when eating.

The medical term for this feeling is globus. Some physicians have used the term globus hystericus to describe this sensation, because it was once thought usually that the description of lump in the throat usually was associated not with organicity, but with symptoms of hysteria.

Liquids vs solids: Patient may report a change in their dietary habits that is associated with perceived dysphagia. Those who complain of the globus sensation often have more difficulty swallowing solids than liquids. Patients with solid food dysphagia are more likely to have disorders of esophageal origin; whereas these who complain of dysphagia for liquids are more likely to have oropharyngeal dysphagia. When patients complain of choking on liquids or solids, a more pharyngeal focused cause is suggested. Whereas those who report dysphagia for liquids \& solids without choking episodes may have a more esophageal focused cause.

Gastroenterologists who support the esophagus as the source of dysphagia may use a decision tree such as the one presented below to assist in diagnosis. Such a decision tree has not been validated against a large number of patients with confirmed diagnosis; however, the concept is useful because the symptoms related to the represented diseases are well known, \& the no. of potential causes for esophageal dysphagia is limited.

Symptoms \& signs that may related to many disease entities. Thus using a decision tree approach based on patient complaints does not provide enough precision to help the clinician establish a diagnosis for Patient with oropharyngeal dysphagia. Gastro esophageal reflux: some Patient complain of episodes of gastro esophageal reflux (heart burn) associated with their complaint of dysphagia. Some Patient describe pain or fullness in the chest associated with their reflux.
Others may have reflux \& dysphagia but may be unaware that they have reflux because the overt symptoms of chest pain or acid taste are not present. Not all Patients describe episodes of reflux unless questioned by the examiner, because they may not relate their episodes to their dysphagia. This is particularly true when Patient complain of globus sensation in the neck, because they might think that reflux in the esophagus could not be related to a problem in the throat. Eating habits: a Patient's report of changes in his/her eating habits may signal the presence of dysphagia, its level of severity, \& its psychological impact.

Complaints that elimination of specific food items from the diet, such as liquids or solids or items that are sticky or crumby, may help the examiner focus the evaluation. Excessive chewing of solid food to avoid a sticking sensation may be more consistent with esophageal disease Vs the pharyngeal focused complaint that liquids always seem to come back through the nose. People/ patients who report excessive time to finish a meal often have dysphagia that requires careful evaluation. Patients who report that they no longer feel comfortable eating in a restaurant because they have to regurgitate or choke should be examined with care. Patient who have experienced marked weight loss or who no longer enjoy the pleasures of eating probably have dysphagia that has reached a high level of severity.

Signs of dysphagia: signs are objective measurements or observations of behaviors that people elicit during a physical examination. In a dysphagic patient who is cooperative, this measurement entails an examination of the cranial nerves relevant to swallowing. Some signs are seen during observation of the Patient eating. Signs \& symptoms may overlap. Example A Patient may complains have liquid going into the nose \& food sticking. Both may be seen by the examiner on the video fluoroscopic swallowing study.

a. The physical evaluation of a Patient may reveal signs that are consistent with dysphagia, such as

b. Drooling from the lip or tongue weakness

c. Poor dentition

d. Loss of strength or range of motion in the tongue, jaw or velum

e. Poor strength or coordination may result in choking on liquids during test swallows or in lack of bolus flow

f. The pt's cognitive status may impact swallowing eg. Failure to chew, talking while swallowing, inattention to the feeding process

Patient who are hospitalized may have more overt medical signs, such as
a. Feeding tubes that are already placed
b. A tracheostomy tube
c. Respiratory congestion after eating 
d. Requirement of excessive oral \& pharyngeal suctioning

e. Eating refusals

f. Under nutrition \& muscle wasting

g. Inability to maintain an upright feeding position

h. An endo tracheal tube

i. Regurgitation of food

The process of evaluation begins with case history, clinical or bedside swallow examination and the instrumental examination. In many assessment protocols, the case history and bedside swallow evaluation are combined. They provide greatest amount of information on the patients eating behavior, language, cognition and oromotor function.

\section{Screening procedure}

Screening procedures provide the clinician with some indirect evidence that the patient has a swallowing disorder. It tend to identify the signs and symptoms of dysphagia such as coughing behaviors, history of pneumonia, drooling, chewing difficulties or the presence of residual food in the mouth. Screening procedures are generally performed at the patient's bedside or in a home or school environment and provide the clinician with increased evidence that the patient needs an in depth physiological assessment.

In infants, children and developmentally delayed adults, certain abnormal behaviors observed during eating are important indicators of the need for in depth physiologic study. These behaviors include rejection of food, food selectivity, gagging, open mouth posture. Two such screening tests in adults are the Burbe Dysphagia Screening Test (BDST) and the screening test proposed by Odderson \& McKenna.

In adults, the Burbe Dysphagia Screening Test (BDST) is used which consisted of a seven items. It checks the presence of one or more items in the test results in failure and then referral for a complete bedside swallow evaluation. The screening items are

I. Bilateral strobe

II. Brain stem stroke

III. History of pneumonia in acute phase strobe

IV. Cough during 303 water swallow or associated with feeding

V. Failure to consume one half of meals

VI. Prolonged time required for feeding

VII. Non-oral feeding programs.

This test is reported to highly valuable in identifying patients are risk for swallowing problems

\section{Bedside examination}

It is designed to define the function of patients lip, tongue, velopharyngeal region, pharyngeal walls and larynx as well as his/her awareness of sensory stimulation. The physiology of some of these structures can be easily assessed at the bedside, while others can only be examined accurately in radiographic or other instrumental study. It consists of following examination.

\section{Review of patient's medical chart}

a. Prior to entering the patients room, the clinician should carefully review the patients medical chart, focusing particularly on the medical diagnosis, any prior or recent medical history of surgical procedures, trauma, neurological damage as well as patients current medications.

b. After defining medical diagnosis, the clinician should immediately consider what physiologic or anatomical swallow disorders that are typical of that diagnoses.

c. History of any respiratory problems should also be identified, including need for mechanical ventilation or tracheostomy tube, the conditions under which they were placed (emergency/planned).

d. Prior history of GI dysfunction should be noted.

e. Prior history of dysphagia from earlier stroke or head injury should be high-lightened even if the patients or his/ her family indicates that the patient returned to oral intake with no apparent difficulty.

f. Medical chart should reveal the patients current nutritional status and the presence of any non-oral nutritional support such as naso-gastric tube.

g. Clinical should also be able to identify the patients general progress as well as prognosis from chart review.

\section{Oromotor examination}

It begins with the examination of anatomic structure of oral cavity including its symmetry and presence of any scar tissue indicating surgical/traumatic damage. The oral examination should note the presence and status of oral secretions, especially the pooling of secretions or excessive dried secretions. In general, the locus of excess secretions in the oral cavity indicates the areas of lesser lingual control or injury. Oromotor examination should then proceed to examination of strength, range of motion and coordination of the lips, tongue and palate for speech and non-speech tasks as well as observation of lingual function and lip closure while the patient produces spontaneous swallows, clinician notes down the frequency of spontaneous swallows.

\section{Respiratory support}

Respiratory support should be defined by counting the rate of breaths per minute. Patients should be asked to hold their breath for a total of $1 \mathrm{sec}$, then 3, 5 and $10 \mathrm{sec}$ and the clinician should observe whether this behavior creates any respiratory distress. Duration of breath hold should be increased as tolerated by the patient. This determines whether the patient can tolerate 
swallow maneuvers or other therapy procedures that increase the duration of apneic or airway closed period during the swallow. Generally patients need to hold the breath of 5 seconds to use swallow manneurs comfortably. The patients coordination of swallowing and respiration should be observed.

\section{Prolonged phonation}

Prolonged phonation on the vowel/o/ should also be examined in terms of both vocal quality and respiratory control used. Clinician should then check whether the patient is able to take an easy inhalation followed by a slow drop of chest and inward motion of abdomen to produce a prolonged vowel on sustained phonation of at least 10 seconds.

\section{Gag reflex}

This is to examine the pharyngeal wall motion as part of the motor response for gag. The pharyngeal wall motion during the gag should be symmetrical. Any asymmetry-unilateral pharyngeal wall paresis.

\section{Laryngeal examination}

a. Series of voluntary tasks will be tested which are as follows:

b. Vocal quality on prolonged /a/ (hoarse, gurgley)

c. Strength of voluntary cough

d. Strength of throat clearing

e. Clarity of / $\mathrm{h} /$ and /a/ during repetitive /ha/

f. Pitch range (slide up and down scale)

g. Loudness range

Cognitive and language characteristics: Through all oromotor testing, the clinician will be examining the patients general behavioral level, ability to discipline his/her own behaviour, and focus on tasks, impulsiveness, ability to respond to commands, etc, should also be tested.

Optimal protocols: De Pippo et al. have proposed other options in place of bedside swallow evaluation. They found that cough or voice change during or directly after drinking 303 of water was sensitive and valid screening tool for aspiration following a stroke. It should be remembered that the clinical swallow assessment with water should be tried only after the findings from patient history and oropharyngeal examination should be taken into account. Patients unable to tolerate their secretions, who have limited attention such as those after a severe stroke or who resist for some other reason may not be the candidates for clinical swallow test.

Dysphagia screening: Prior to bedside swallow, use of dysphagia screening test may be appropriate. This is usually done by speech language pathologist but may also be done by a nurse trained in the procedure. Two such screening tests are the Burbe Dysphagia Screening Test (BDST) and the screening test proposed by Odderson \& McKenna. The BDST consists of a seven item test. Presence of one or more items in the test results in failure and then referral for a complete bedside swallow evaluation.

The screening items are

a. Bilateral strobe

b. Brain stem stroke

c. History of pneumonia in acute phase strobe

d. Cough during 303 water swallow or associated with feeding

e. Failure to consume one half of meals

f. Prolonged time required for feeding

g. Non-oral feeding programs.

These 2 tests are reported to highly valuable in identifying patients are risk for swallowing problems.

\section{Dye test}

Also known as Blue dye test may be used to determine the presence of aspiration in a tracheostomized patient. A few drops of methylene blue or vegetable coloring are placed in the mouth, tracheostomy cuff is deflated, and the tracheostomy tube is deep suctioned for secretions that may have been resting on or above level of cuff. The patients tracheostomy tube is deep suctioned and looking for evidence of dyed material in airway. This may not detect trace amounts of aspirated materials.

\section{Auscultation: Chest and cervical}

Placing a stethoscope over various parts of airway provide indirect evidence of aspiration. Through this, he can listen to airflow during normal breathing, swallow sound. It determines whether other tests are needed.

\section{Clinical Swallowing Examination (CSE)}

The clinical swallowing examination allows a circumscribed exploration of patients muscle function, sensation and airway protective functions. This CSE allows the clinician to develop management program for the patient and to determine the necessity of further instrumental assessment. The clinical swallowing examination protocol includes the following:

\section{CSE 1 Mental status}

We know that there is interdependence between safe swallow function and cognitive and behavioral factors such as attention, memory, judgement, reasoning, orientation and sequencing skill. In patients with head injuries, the frequency of swallowing disorders was found to decrease as patient's scores on level of cognitive function scale improved [2].

During the interview, clinician should be vigilant for indications of reduced mental function.

a. Are the clothes clean or blotched with food particle? (Subtle questions) 
b. Is there an evidence of appropriate attention to cleanliness and hygiene? (Subtle questions to cleanliness and hygiene? (Subtle questions).

c. Is the individual attending to the questions and answering appropriately?

d. Is there a drift during the session?

e. Is the caretaker/spouse acting as a surrogate in the interview without invitation...... etc

Many scales have been developed for measuring and monitoring mental status eg.

a. Glassgow coma scale. It is scored for 3 behaviors, eye opening verbal response and motor response. The score range from 3 (severe coma) to 15 (full awareness)

b. The Ranchos Losnmigos scale.

c. All these scales tell us about degree of cognitive impairment.

\section{CSE 2 Speech/Articulation}

Here the clinician makes a gross determination of

a) Precision of articulation i.e. speech intelligibility, look for the $\%$ speech intelligible

$100 \%$ - normal

$>50 \%$ - moderate

$35-50 \%$ - severely affected

$<35 \%$ - very unintelligible

b) Rate - normal; slow; accelerated

c) Predominant error- Check whether distortion/ omission/substitutions present. Distortions are more likely to be present in neurogenic speech disorders.

\section{CSE 3: Respiratory function}

Here the respiratory subsystem is assessed which includes -

a) Volitional cough: Ask the patient to take a breath and produce as great a cough as possible. Check whether he is able to cough or not if not see for the presence of forced expiration, throat clearing or hawking. (Hawking: audible effort to force out the phlegm from throat).

Also check for productive cough (transport of material from lower airways.

Check for loudness (normal, weak/audible or very weak/ inaudible).

b) Sustained expression while counting: Ask the patient to inhale as deeply as possible and with a single breath, count as high as you can. The score is derived from the number reached when patient counts aloud on a single exhalation after maximum inspiratory effort.

c) Index of pulmonary dysfunction: Smeltzer, Skurnick, Toroiano, Cook, Duran and Lavietes (1992) employed an index sensitive to pulmonary dysfunction. The possible range of scores 4 (normal maximal expiratory pressure to11 (poor maximal expiratory pressure) [3,4].

\section{CSE 4: Voice/resonance}

For assessing this, clinician will rely on connected speech and check for normal/hoarse/ harsh/hypophonic/ aphonic or wet dysphonic/hypernasal.

\section{CSE 5: Position}

Clinician will observe the patients habitual body and head position and examine the patients adaptations or apparatus used to assist in support. The clinician then will attempt to elicit alterations in body and head positions.

\section{Body position}

a. Leaning with self support.

b. Supported by apparatus.

c. Reclined.

\section{Head positioning}

a. Flexion.

b. Extension.

c. Head turned to left/right

Eliciting changes in position during the clinical examination allows the examiner to probe for the patients capacity to change the position or posture later in assessment process. Repositioning the head and trunk has been shown to cause changes in biomechanics of swallowing.

\section{CSE 6: Lip sensation/strength/seal}

The clinician will assess the sensation, strength and range of motion of the lips. The equipment needed includes cotton lip applicators and a tongue blade.

a. Ask the patient to close his/her eyes and respond either verbally or by raising a finger or hand in response to stimulus placed on the lip and its marging (light momentary brush over).

b. Checking drooling is present/not.

c. Note down the habitual oral position ie open/closed.

d. Lip strength can be assessed by asking the subject to purse his/her lips with as much pressure as possible and ask him to lift the upper and lower lip with tongue blade along its entire length.

\section{CSE 7: Mouth opening}

a. The patient is asked to open his mouth as widely as it will open and see whether it is normal/reduced mouth opening (approximate mouth opening: $1 \mathrm{~cm}$ ).

b. This is assessed because patients with small mouth openings may have great difficulty placing even small 
volumes of food in their mouth. The amount of time and effort need to take in enough food to maintain nutrition lead these patients to abandon oral solid foods in favour of liquid diets that are easily consumed by straw.

\section{CSE 8: Muscles of mastication}

Masseter and temporalis: They act to move the mandible to a closing position. With the jaw muscles relaxed, ask the patient to clench down on a tongue, blade placed along the length of the molars on the right or left side of oral cavity. Palpate and note down the bulging and firmness.

Lateral and medial pterygoid: Check for protrusion and lateralization by applying resistive pressure on the other side with other hand.

Check for pain while performing the above mentioned tasks, see whether the pain is unilateral or bilateral also.

\section{CSE 9: Dentition and periodontum}

Clinician assesses the patients dentition prosthetic dentition and gingiva. The equipment needed includes a penlight, gloves and tongue depressor - we have to make a note of existing teeth and missing teeth in the tooth chart - also indicate the condition of existing dentition.

Removable prosthetics: While inspecting the mouth, make note of removable partial/complete dentures. A partial denture replaces one or more teeth in one arch. A complete denture replaces most or all of the teeth in an arch. Indicate where the prothesis are in place and note their condition with the patients mouth wide open, grasp the denture and tug on it to determine firmness - check for the presence of food particulate or plague on the surface of the denture.

\section{CSE 10: Salivary flow/appearance of oral mucosa}

Check whether the salivary flow is normal or any hyposalivation is present.

Check for the appearance of oral mucosa i.e. surface of the tongue appears severely dry, tongue with cracks and fissures etc.

\section{CSE 11: Oral/pharyngeal gag}

Have the patient open his/her mouth as you probe the surface of the tongue, faucial pillars and posterior pharyngeal wall. Initially apply light touch and probe for the response. If no response, apply enough pressure.

\section{CSE 12: Tongue movement/strength}

a. Request the patient to open his/her mouth and observe the tongue at rest and during protrusion. Check whether the tongue is normal/atrophied/deviated/dyskinesia.

b. Also check for nasal respiration.

c. A patient with normal strength and coordination should be able to breath many cycles with the cheeks puffed. Even if the push the cheeks in both hands, air should escape thro lips. This task tests the posterior seat of oral cavity.

d. Tongue strength should be tested with isometric exercises. See whether strength is normal/reduced.

e. Tongue range of motion can be checked by requesting the patient to move the tongue tip along the entire length of maxillary and mandibular buccal sulci. Also ask the patient to stroke over the surface of hard palate.

\section{CSE 13: Velar elevation}

Ask the patient to say /ə/ or yawn and check for velar elevation.

\section{CSE 14: Volitional swallows/laryngeal elevation}

a. Place the ring, middle and index fingers along the entire surface of throat with the index finger in the superior position. Situate your finger so that the thyroid notch is nestled between ring and middle fingers. Here index fingers should be resting on suprahyoid muscles. Request the patient to swallow with the fingers lightly resting upon the suprahyoid muscles. There may be a moment of delay as the patient collects saliva within oral cavity and prepared to swallow.

b. Patients with xerostomia - no enough saliva to initiate dry swallows.

c. Apraxia - no coordinated swallow

d. Cognitive impairment - unable to swallow on common.

e. The sublingual musculature pull away from index finger as the suprahyoid muscles contract. During elevation, the thyroid notch should move to a point above the middle finger and at the apex of the swallow, may come in contact with the inferior margin of the index finger. A swallow following this pattern is normal.

\section{CSE 15: Food and liquid swallows}

The clinician will elicit swallows by presenting food and liquid of varying volumes and consistencies to the patient. The clinician will observe and record signs and symptoms that are exposed during the session. As a basic guideline, the clinician should hold safety as the highest priority. Clinician should be sure of the following:

a. Alertness

b. Cough: strong cough, weak cough

c. Accordingly we can introduce any material in the patients mouth.

d. Order of presentation should be taken into account. The initial delivery should be of and consistency that will be the easiest for the patient to consume. Graded activities should be done eg. Slowly increasing the volume.

e. Enter the maximum amount of food or liquid presented with a particular material (listing the volume is also important). 
f. Timing of the onset is dependent on the materials presented with longer period necessary as the hardness of the food increases. The elapsed time should begin as the food enters the mouth and end as the larynx elevates for pharyngeal swallow.

g. Clinician should count the number of swallows per bolus delivery by recording the number of elevations of the larynx. When multiple swallow occurs, the clinician should query the patient regarding the presence of food sticking. If able, have the patient point to the perceived location of the residue. Accordingly instrumental techniques is indicated.

h. Oral signs: Clinicians should look inside the patients oral cavity following each food presentation in those patients who report oral stage difficulties. Attention should be given to all cervices and pockets where food can get accumulated.

i. Airway signs - note the presence of wet dysphonia and frequent cough (liming and strength).

\section{Instrumental Techniques for the Study of Swallowing}

\section{Introduction}

A number of imaging and non-imaging instrumentation procedures have been used to study various aspects of normal and/or abnormal swallow physiology. Each procedure provides some pieces of information on oropharyngeal anatomy or swallow physiology. It is important that the clinician be familiar with the types of information, each procedure provides information about swallowing and basic methodology for each procedure. These instrumental procedures include

a. Imaging studies.

b. Non-imaging studies.

\section{Imaging studies}

Several technologies can be used to image the oropharyngeal region which include ultra sound, video endoscopy, video fluroscopy, pulse oximetry, scintigraphy, CT and MRT, esophagoscopy/gastroscopy.

\section{Ultra sound}

Ultra sound uses high frequencies sounds ( $>2 \mathrm{MHz}$ ) from a transducer held or flexed in contact with skin to obtain a dynamic image of soft tissues. As ultrasound does not penetrate bone, its use is limited to the soft tissues of oral cavity and parts of the oropharynx. Here a handheld transducer is placed submentally and is rotated 90 degrees. It is used to observe tongue function and to measure oral transit times as well as motion of hyoid bone. Real food can be used here.

\section{Videoendoscopy (FEES)}

It has been used increasingly in recent years to examine the anatomy of oral cavity and pharynx and to examine the pharynx and $\mathrm{lx}$ before and after swallowing. First described by Langmore et al. It requires the passage of a fiberoptic laryngoscope into nares, over the velum to a position above the epiglottis. After placing the endoscope, examiner notes the anatomic structures and observes the functions of the velum, epiglottis and larynx using sustained phonation or repeating coca cola. Trial dry swallows are prompted to elicit laryngeal elevation.

Specific amounts of liquids and food consistencies treated with food dye are viewed as they pass the pharynx and larynx. During the time of airway closure, swallow cannot be visualized as the pharyngeal walls contract over the bolus, collapsing the lumen over the endoscope (whiteout phase). Monitoring of the bolus is only possible before and after pharyngeal swallow. Video camera monitors the bolus as it enters the view from oral cavity to pharynx.

It can be used to determine sensory dysfunction in dysphagic patients. To perform the test, an air pulse generator is used to send a pulse of air through a port in a specially designed flexible nasopharyngoscope. Air pulses can be delivered to the supraglottic larynx and pharynx areas. Using a calibrated puffs of air, sensory thresholds can be determined using one of the psychophysical testing methods. The twitching response of the mucosa suggests the sensory awareness of stimulus.

\section{Scintigraphy}

It is a nuclear medicine test in which the patient swallows measured amount of radioactive substance (technetium-99m) combined with liquid/food. A special gamma camera records images of the organs of interest over time. This can also be used to identify aspiration, quantify aspiration over short/long periods of time. It can also be used to calculate transit time and residual pooling of a bolus before and after treatment. If there is no aspiration and reflux is suspected, the patient is rescanned every 15-20 min for several hours to detect reflux.

\section{Pulse oximetry}

A relatively new approach to monitor swallowing and possibly detecting aspiration is pulse oximetry. It is based on the principle that reduced and oxygenated hemoglobin exhibit different absorption characteristics to red and infrared light emitted from a finger/ear probe. Pulse oximetry measures oxygen desaturation of arterial blood, a condition which is thought to occur as a result of aspiration. It is non-invasive, simple may be repeated often but does not provide diagnostic information to formulate treatment plans. It offers information regarding presence and possibly severity of aspiration.

\section{Videofluroscopy}

Because swallow is a dynamic and rapid process, videofluroscopy is particularly well suited to the study of this physiologic function. The term cookie swallow has been in the past but this does not describe the procedure adequately.

Modified barium swallow has two purposes: To define the abnormalities in anatomy and physiology causing the patients symptoms. To identify and evaluate treatment strategies that may 
immediately enable the patient to eat safely. Baruim swallow test can be used to examine oral transit times, structural competence of oesophagus, particularly lower two thirds of oesophagus very well.

\section{Test}

Under fluoroscopic observation, controlled by the radiologist, the patient ingests barium coated boluses or liquid barium of varying consistencies.

Placement of food in the patients mouth: Generally food is placed in the patients mouth on a disposable plastic spoon. If a patient has a bite reflex, a heavier plastic spoon is more appropriate. For infants, bottle and nipple may be used.

Type and amount of material used: At least three consistencies of material are used in the modified barium swallow to investigate patient complaints of variable swallowing ability. Thin liquid barium (as close to water as possible), barium paste (chocolate pudding mixed with esophatrast) and the material requiring mastication (a cookie coated with pudding mixed with esophatrast). At least two swallows of each material are given in following amounts: $1 \mathrm{ml} 3 \mathrm{ml}, 5 \mathrm{ml}, 10 \mathrm{ml}$ and cup drinking of thin liquid; $1 / 3$ teaspoon of pudding and a fourth of a small Lorna Roone Cookie coated with barium [2]. Volume of liquid is increased until or unless patient aspirates.

Positioning the patient: Often the most difficult and time consuming part. No. of chairs for positioning patients during the radiographic study have been designed. A patient who is mobile and able to sit without a backrest can be seated on the horizontal platform attached to fluoroscope table and raised/lowered to desired height. Most machines are fitted with handles so that the patient can stabilize his/her position. Some fluoroscopy machines will not accommodate wheel chairpersons or persons on cart. If permitted, they are positioned on a cart with the head of cart elevated to at least 90 angle.

Focus of fluoroscopic image: The fluoroscopy tube should focus on lips anteriorly, hard palate superiorly posterior pharyngeal wall posteriorly and the bifurcation of the airways and oesophagus inferiorly. Many fluoroscopy machines permit image magnification.

Measures and observations to be made: lateral view: This permits a number of measures and observations critical to the identification of patients anatomic/physiologic swallowing disorder. It helps in measuring oral, pharyngeal and oesophageal transit time. This permits identification of the location of the bolus as it moves along upper aerodigestive tract from anterior superior to posterior inferior. It permits the analysis of patterns of lingual movement, estimate of amount of material aspirated per bolus, as well as the reason for aspiration. The timing of aspiration relative to triggering of pharyngeal swallow is also best examined.

The eight step scale maybe quite useful to monitor changes in a patients ability to control aspiration material.

I. Does not enter airway

II. Remains above folds / ejected from air way

III. Remains above folds / not from air way

IV. Contact folds / ejected from air way

V. Contact folds / not ejected from air way

VI. Pass below folds / ejected into lx or out of air way

VII. Passes below folds /not ejected despite effort

VIII. Passes below folds / no spontaneous effort to eject.

Posterior anterior view (P-A view): P-A view is helpful in looking at asymmetries in function, particularly of pharyngeal walls and vocal folds and in viewing the residual material in the valleculae and in one or both pyriform sinuses. It provides vocal fold movement picture too.

\section{Other radiographic tests for dysphagia}

Upper gastro intestinal series: The single contrast esophagram study fills and distends the lumen with thin liquid barium. Intrinsic mural irregularities and masses and extrinsic impressions are visible. An air contrast study provides the same information but allows a more detailed view of mucosa. For an air contrast barium study, the patient ingests effervescent crystals followed by thick barium. A barium swallow has both dynamic and static components. The dynamic portion, fluoroscopy can be recorded on tape (video fluoroscopy cine radiography) for later review. The static portion is recorded on a series of rapid still frames.

The barium swallow can identify intrinsic and extrinsic pathology. Intrinsic abnormalities include tumors, cricopharyngeal dysfunction, aspiration of barium into airway or reflux into nasopharynx, diverticulas webs and esophageal dysmotility. Extrinsic masses such as cervical osteophytes and an enlarged thyroid gland maybe visualized directly or suspected by their effect on the barium column.

The subjective location of dysphagia does not always correspond to anatomic location of pathology. Therefore, the barium study when used to evaluate dysphagia should extend as low as the gastric fundus or cardia. The upper gastroesophageal series evaluates the stomach and relaed areas. Obstruction or dysfunction of these areas may cause or contribute to esophageal dysfunction (eg. GERD). Thus the transitional barium swallow evaluates the upper aerodigestive tract between oral cavity or oropharynx and gastric fundus or cardia. It is not intended to identify swallow dysfunction or to dictate treatment as in the modified barium swallow.

Computer tomography and magnetic resonance imaging: They are used to delineate the anatomy of a particular region of the head, neck or other components of the upper aerodigesture tract. The most common use is to identify a site of lesion such as 
cerebrovascular accident within the central nervous system or to delineate the extent of an intra/extra luminal space occupying system. In general, CT offers direct axial and coronal images that better define bony anatomy.

MRI better delineates the soft tissue in saggital, coronal and axial planes but takes longer to complete the images and thus is prone to motion artifact. High speed MRI such as fast low angle shot (FAST) or echo planar imaging, has allowed a dynamic analysis of the pharyngeal phase of swallowing that was impossible with conventional MRI. The pharyngeal oral cavity, laryngeal lumen and musculature can be evaluated during motion, allowing the assessment of swallowing mechanism. During FAST MRI, images are obtained as a bolus containing contrast substance is so allowed. Here temporal and spatial resolution is poor, but no exposure to radiation.

Non imaging techniques: They provide a variety of types of information about swallowing but do not results in pictures of the swallowing process or the food being swallowed. Instead, most result in amplitude over time displays of the swallow parameters being examined.

Electromyography: EMG of muscles involved in swallowing can provide information on the timing and relative amplitude of selected muscles contraction during swallowing. The electromyogram is recommended to ascertain the presence of specific nerve or neuromuscular unit deficit such as that accompanying vocal fold paralysis or to elucidate or corroborate the presence of systemic myopathy or degenerative disorders. The goals of a laryngeal EMG are to detect normal from abnormal activity and localize and assess the severity of a focal leision by determining whether there is neuroaphraxia (physiological nerve block or focal injury with intact nerve fibers or axonotmesis) damage to nerve fibers leading to complete peripheral degeneration. Laryngeal EMG can evaluate prognosis too.

The thyroarytenoid muscle is approached by insertion of a monopolar or concentric electrode through the cricothyroid ligament midline $0.5 \mathrm{~cm}$ to $1.0 \mathrm{~cm}$ then angled superiorly $45^{\circ} \mathrm{C}$ and laterally $20^{\circ} \mathrm{C}$ for a total depth of $2 \mathrm{~cm}$. The cricothyroid muscle is reached by inserting the electrode $0.5 \mathrm{~cm}$ off the midline, then angling superiorly and laterally 20 degrees towards the inferior border of thyroid cartilage. Laryngeal EMG help differentiating neurological vocal cord paralysis from laryngeal joint injury. It may also confirm the diagnosis of joint dislocation. The 3 areas of interest for electro diagnostic evaluation of swallowing are the laryngeal sphincter, the sensory ability of the supraglottic larynx and pharynx (indirectly evaluated through cricothyroid muscle function) and the cricopharyngeal sphincter. EMG has several pitfalls like precise site of lesion cannot be determined except whether it involves vagus nerve (SLN and RLN). PCA is difficult to localize through this. Systemic neuromuscular diseases cannot be differentiated from focal lesions.
Direct laryngoscopy: Endoscopy of the upper aerodigestive tract is recommended to rule out biopsy or neoplasm that may be suspected to be the cause of dysphagia or odynophagia. Occasionally, the endoscopy may be part of the treatment as in those patients requiring injection of the paralyzed vocal fold, injection of BOTOX or dilation of the oesophagus for the treatment of cricopharyngeal achalasia or strictures.

Electroglottography: EGG is designed to track vocal fold movement by recording the impedance changes as the vocal folds move toward away from each other during phonation. This equipment can be modified to track laryngeal elevation which can be useful in determining the onset and termination of a pharyngeal swallow and in providing biofeedback to an extent and duration of laryngeal elevation during the swallows in which the patient is attempting to improve these swallow parameters.

Esophagoscopy/Gastroscopy: Dysphagia and odynophagia are common indications for upper Gastro intestinal endoscopy and may be performed as the initial test in the evaluation of these disorders. The esophagus is intubated under direct visualization of hypopharynx. The endoscope is usually advanced through the upper esophageal sphincter which appears as a slit like opening in the cricopharyngeous muscle at about $20 \mathrm{~cm}$ from the incisor teeth. The entire length of the esophagus is indirect view of the endoscope until its termination at the gastroesophageal junction which lies at the diaphragmatic hiatus.

The esophagus is usually closed at gastroesophageal junction but this is easily distended with air insufflations. This allows the endoscope to easily advance through the lower esophageal sphincter into the stomach. Upper GI flexible endoscopy is the most specific test for identifying esophageal complications of GERD, esophageal ulcer, infectious disorders and neoplasms. It is useful in defining the cause of disease in those patients with solid food dysphagia.

Cervical Auscultation (Listening to and recording the sounds of swallowing): Recording the sounds produced during the swallow by placing a small microphone or accelerometer on the surface of the patients neck at various locations has identified some repeatable sounds produced across normal subjects (Hamlet, Nelson \& Patterson, 1990; Patterson, Hamlet, Fleming \& Zones, 1992). Another method for listening to the sounds of swallowing is to apply a stethoscope to the patients neck. The ability to distinguish normal from abnormal sounds or a define the meaning of the sounds produced during swallow by patients with swallowing disorders has not been determined. It needs further research.

Cervical auscultation (Sounds of respiration): This can define inhalatory and expiratory phases of the respiratory cycle as well as the moment when the pharyngeal swallow occurs and in which part of the respiratory cycle, swallowing occurs. If the secretions are in the airway before or after the swallow, these will also be heard, as will any changes in secretion levels 
before and after the swallow. Information on secretion levels and changes in these levels before and after the swallow may be indicators of aspiration.

Esophageal Ph monitoring: Prolonged (24hour) esophageal $\mathrm{pH}$ monitoring is the most reliable test for diagnosing GERD. Twenty four hour $\mathrm{pH}$ monitoring is usually done following an overnight fast. The $\mathrm{pH}$ catheter is inserted trans-nasally into the esophagus. Standard placement of the distal probe is at a position that is approximately $5 \mathrm{~cm}$ above the proximal border of the lower esophageal sphincter. It is ultimately attached to recording device. Patients are asked to record in a diary or in the recording device, the times that they eat, sleep or perform any other activities. More importantly, patients will be asked to record any type of discomfort that they have including all the symptoms they experience. This information will be used to correlate the $\mathrm{pH}$ at the time a symptom or activity took place and a symptom index can be calculated. $\mathrm{pH}$ should be less than 4 in normal individuals.

\section{Manometry}

a. Esophageal manometry.

b. Pharyngeal manometry.

Pharyngeal manometry: The response of the oropharynx to swallowing has two components. The first is compression of the catheter against the pharyngeal wall by the tongue which results in a high sharp peaked amplitude pressure wave. This is followed by low amplitude, long duration wave, which reflects the initiation of pharyngeal peristalsis. A rapid, high amplitude pressure upstroke ending in a single sharp peak, followed by a rapid return to baseline is produced by contraction of middle and inferior pharyngeal constrictor muscles to provide mid pharyngeal response to swallowing.

The pharynx is not radially symmetrical and therefore, the measurements obtained during standard manometry vary with the catheter placement. Nonetheless, measurements of intrabolus pressures during the pharyngeal phase of swallowing may predict which patients will respond to a surgical myotomy. A polyvinyl catheter, a thin tube about $35 \mathrm{~cm}$ long made of a flexible polyvinyl material and constructed with multiple pressure sensors, is passed trans-nasally and the patient is instructed to perform a series of wet and dry swallows. Lower esophageal sphincter pressure is measured at baseline and in response to a swallow. Lower esophageal sphincter pressure is measured as a step up in pressure from gastric baseline referenced as atmospheric.

Complete lower esophageal sphincter relaxation with swallow is demonstrated by a decrease in pressure to gastric baseline for approximately 6 seconds. Basal, upper esophageal sphincter pressures can be identified as a rise in pressure above the esophageal baseline. Due to the asymmetry of the upper esophageal sphincter, this is normally $50-100 \mathrm{~mm} \mathrm{Hg}$ depending on duration of the pressure sensor (whether lateral or anterior/ posterior). Evaluation of upper esophageal sphincter relaxation and correlation of sphincter relaxation with pharyngeal contraction is obtained by instructing the patient to perform a series of wet swallows.

Acoustical analysis: This is more accessible for speech language pathologist- done or useful in individuals with mild dyphagia. Here we will be measuring the swallow sound and comparing it with the normative developed

Mechanism of the production of glottal sound: It is known that arytenoids closure, true vocal folds and laryngeal elevation provide the basis for laryngeal closure during swallowing. The mechanism of arytenoids closure and true vocal cord closure valves the larynx shut. In valving the airway during the period of deglutition apnea, the sub-glottal sound is pressurized. When the cords apart, there is a release of air and there is a rapid increase in airflow and the release of this is often audible. Shaker et al 1990 show that the simple closure at the level of larynx may not be sufficient to cause the glottal release sound. They say sudden removal of the valve coupled with elastic recoil of the lungs provides us with the environment required to produce the glottal release sound.

Table 1:

\begin{tabular}{|c|c|}
\hline Symptoms & Sign \\
\hline Difficulty chewing & $\begin{array}{l}\text { Food spills from lips; excessive mastication time } \\
\text { of soft food; poor dentition; tongue; jaw or lip } \\
\text { weakness. }\end{array}$ \\
\hline $\begin{array}{l}\text { Difficulty } \\
\text { initiating swallow }\end{array}$ & $\begin{array}{c}\text { Mouth dryness (xerostomia); lip or tongue } \\
\text { weakness }\end{array}$ \\
\hline Drooling & Lip or tongue weakness, infrequent swallow \\
\hline $\begin{array}{l}\text { Nasal } \\
\text { regurgitation }\end{array}$ & $\begin{array}{l}\text { Bolus enters or exits the nasal cavity as seen on } \\
\text { radiographic swallowing study }\end{array}$ \\
\hline Swallow delay & $\begin{array}{c}\text { Radiographic study identifies transport beyond } \\
\text { normal standard }\end{array}$ \\
\hline Food sticking & $\begin{array}{c}\text { Radiographic study identifies excessive residue } \\
\text { in mouth, pharynx, or esophagus after completed } \\
\text { swallow }\end{array}$ \\
\hline $\begin{array}{l}\text { Coughing \& } \\
\text { choking }\end{array}$ & $\begin{array}{c}\text { Coughs on trail food attempts; material enters } \\
\text { the air way on radiographic study }\end{array}$ \\
\hline $\begin{array}{l}\text { Coughing when } \\
\text { not eating }\end{array}$ & $\begin{array}{l}\text { Radiographic study shows aspiration of saliva or } \\
\text { lung abnormality }\end{array}$ \\
\hline Regurgitation & $\begin{array}{c}\text { Undigested food in mouth; radiographic } \\
\text { study shows food returning from esophagus } \\
\text { to pharynx or mouth mucosal irritation on } \\
\text { endoscopy }\end{array}$ \\
\hline Weight loss & $\begin{array}{l}\text { Unexplained weight loss; measurement of weight } \\
\text { is below ideal standard }\end{array}$ \\
\hline
\end{tabular}

Recording of the glottal release sound: Subjects should be seated in a back straight chair. The cervical region should be cleansed with a alcohol swipe. The microphone should secured to the cervical region at the midline of the cricoid cartilage using a single sided surgical tape such that there is no air escape around the microphone. Then it should be attached to the preamplifier which will feed the acoustical signal directly to the computerized 
speech science lab (CSL 4300, Kay Elemetrics) or any software which has spectrographic analysis.

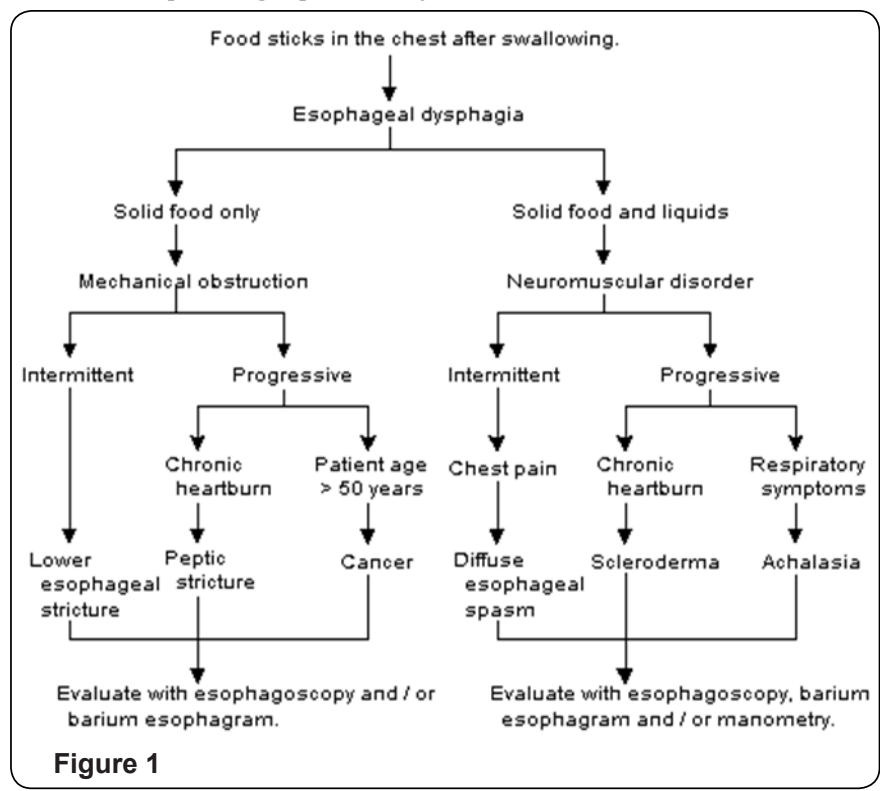

Subject should be given trials of water swallow to allow the investigator to set the optimal recording level. Subjects should be requested to swallow in one complete action. Recording of the swallow sound commenced upon the lip cup contact. Recording ceased post swallow after the laryngeal movement has been visualized and the individual brought the cup back to the rest position. Recording should be done directly on to the disk. Each swallow should be displayed as a raw acoustic signal and linked by curser and its narrow band spectrogram. The beginning of swallow sound was marked by combination of auditory feedback and visual inspection of the spectrogram. Parameters which need to be measured include frequency, intensity and duration. This needs to be compared with norms of particular age with varying thicknesses of fluid as shown in the Table $1 \&$ Figure 1.

\section{Nutritional concerns and assessment in dysphagia}

Successful management of dietary needs in dysphagic patients requires the efforts of all involved caregivers. The particular responsibility of the dietitian is to assess a patient's nutrition and hydration needs and to translate these needs into a diet that meets restrictions imposed by the team, i.e., oral feeding, oral feeding with compensatory safeguards or facilitators, enteral feeding, or combined enternal and oral feeding. As these needs change, the dietitian should be involved to make the transition as effective as possible. Another important responsibility of the dietitian is to make the diet prescribed as appealing and palatable to a patient as possible.

Both screening and comprehensive nutritional evaluations require consideration of a patient's anthropometric characteristics, dietary intake, relevant clinical and physical findings and socioeconomic considerations. Comprehensive assessment will elaborate each of these areas, for example, dietary intake will be assessed in terms of calories, protein, vitamins, minerals and fluid and evaluated for adequacy based on the patient's individual needs.

Indications: Nutritional assessment should be considered when a patient's mean of feeding has been altered, when such a change is anticipated, or when there are concerns about the amount and / or nutrition/hydration value of a patient's diet. Populations considered here are those most likely to benefit from a team evaluation, for example, patients for whom there are a possibility of safe oral feeding and whose mentation level is judged adequate for at least a minimal level of cooperation with team objective. Age groupings of patients have additional significance. For example, one can expect the CVA (cerebral vascular accident) population to be older, in general, and the head trauma population to be younger. Such differences not only have implications for dietary needs, but also may influence a patient's eligibility for funding sources, which support nutritional needs.

In some populations, a change in eating status is anticipated, and dietary needs can be addressed prior to the expected event. In other populations, the need for nutritional assistance is acute and unanticipated. Patients who are apparently well and then experience a sudden insult, i.e., CVA or trauma are examples of this kind of population. Other patients may be maintaining adequate nutrition / hydration but have few reserves to cope with new or unexpected problems that compromise their nutritional well being. In such cases, changes in nutrition / hydration status are not entirely unanticipated. Careful monitoring to assure prompt intervention is necessary.

Red flags' for formal dietitian consult: Significant changes in weight trends and hydration status in a patient signal the need for a comprehensive nutritional evaluation. Guidelines for determining the severity of unintentional weight loss are presented in Table 2. In general, a person losing $10-20 \%$ of his or her usual weight may sustain moderate impairment, whereas a loss of greater than $20 \%$ of usual weight indicates severe impairment. In both situations, thorough elaboration of the cause of weight loss will be required.

Table 2: Evaluation of weight change.

\begin{tabular}{|c|c|c|}
\hline Time & $\begin{array}{c}\text { Significant Weight Loss (\% } \\
\text { of Change) }\end{array}$ & $\begin{array}{c}\text { Severe Weight Loss (\%) } \\
\text { of Change) }\end{array}$ \\
\hline 1 week & $1-2$ & $>2$ \\
\hline 1 month & 5 & $>5$ \\
\hline 3 months & 7.5 & $>7.5$ \\
\hline 6 months & 10 & $>10$ \\
\hline
\end{tabular}

Red flags for sub optimal hydration include rapid weight loss (a 48 hour weight loss of 4 pounds can mean a negative fluid balance of 2 liters), complaint of thirst, skin turgor changes, decreased urination, a rising blood-urea-nitrogen level (BUN) in the absence of other renal indicators, and an increased serum sodium level (hypernatremia). Patients with thin liquid dysphagia may be at particular risk for alterations in hydration status. They will have difficulty augmenting fluid intake to 
compensate or increased fluid losses due to secondary illness and are also more vulnerable to other fluid-depleting conditions (i.e., fever, diarrhea, or increased perspiration related to physical exertion or heat).

Nutritional assessment: As noted, careful monitoring of patient's nutritional status can and should be undertaken by members of dysphagia team and other caregivers. However, expedient referral to the dietitian is indicated when there is any question regarding the patient's ability to maintain adequate nutrition / hydration, safely, via the current mean of food intake. Components of the dietitian's examination include the following.

Anthropometric data: As indicated, a primary cue for dietary referral is weight change. Appropriate weight range is impacted by gender, age, height and frame. The patient's usual weight any change in this amount, over what period of time, and whether any change was intentional must be determined. If weight loss is too rapid, and in particular if it is associated with inadequate protein intake, it may adversely impact the body's immune function. The ability to resist disease and infection is compromised. The patient's energy level and ability to participate in the prescribed rehabilitation program may also be affected. Presented in Table 3 are guidelines for interpreting nutritional status based on percent of ideal body weight (IBW) and percent of usual body weight (UBW).

Table 3: Evaluation of nutritional status based on a percentage of weight.

\begin{tabular}{|c|c|c|}
\hline & $\begin{array}{c}\text { \% Of Ideal Body } \\
\text { Weight }\end{array}$ & $\begin{array}{c}\text { \% of Usual Body } \\
\text { Weight }\end{array}$ \\
\hline Mild malnutrition & $80-90$ & $85-95$ \\
\hline Moderate Malnutrition & $70-79$ & $75-84$ \\
\hline Severe Malnutrition & $0-69$ & $0-74$ \\
\hline
\end{tabular}

A value of less than 3.5 to 3.2 is suggestive of the patient being nutritional risk. Other laboratory tests provide a more sensitive indicator of current protein status but these may require special ordering procedures. Laboratory values that are both commonly available and useful for evaluating hydration status are serum sodium and blood urea nitrogen. Elevated values are typical in the dehydrated patient. Additionally, albumin will be elevated in dehydration. One needs to remain mindful that hypo albuminia may be masked by mild to moderate dehydration, resulting in a falsely normal appearing albumin value due to hemo concentration. In dehydration, low urine output will occur as the body seeks to conserve fluid.

Nutrition history: The patient or caregiver is instructed to record the time food or drink is consumed, the amount consumed, and a description of the food and how it was prepared, i.e., steamed, fried, broiled. The amount of food should be described using standardized measurements. The patient is also asked to note if this is a typical meal pattern and, if not, what is different. Any nutrient label information concerning calories and protein per serving size should also be included in the report. It is important to appreciate that merely recording one's dietary intake may alter the usual pattern of intake.
Additional measures obtained by the dietitian are a recent (last 24 hours) food intake record is recalled by the patient (referred to as a '24-hour recall') and a food frequency list, which describes how often the patient has had different types of foods over a recent time period. From all measures considered, the dietitian will compare the patient's dietary intake to standard referents of dietary requirements. For example, the USDA

Food Guide Pyramid recommendations for daily dietary requirements include:

a. 6-11 servings from the Bread, Cereal, Rice and Pasta Group (provides the dietary base)

b. 2-4 servings from the Fruit Group

c. 3-5 servings from the Vegetable Group

d. 2-3 servings from the Milk, Yogurt and Cheese Group

e. 2-3 servings from the Meat, Poultry, Fish, Dry Beans, Eggs, and Nuts Group.

Subsequent monitoring of weight, laboratory values, and the patient's global sense of well being will assist in fine tuning the nutrition goals.

Clinical and physical findings: It is important to stress that oral cavity structures and their functional integrity impact both how and what type of nutrition a patient may be able to manage. For example, the ability to chew to a ground or puree texture will determine the texture(s) of food that can be offered to the patient. Also important to the nutritional evaluation is the patient's level of physical activity. If activity is very sedentary, the patient's energy need and number of calories required will be low, necessitating the selection of nutrient dense food. This is to ensure nutritional adequacy of protein, vitamins and minerals without excess weight gain that would further impact mobility.

Concurrent chronic conditions, such as diabetes, coronary artery disease, renal and pulmonary impairment that may have pre-existing diet management in place, must also be noted. A cardiac patient's low diet may be inappropriate if the patient is unable to consume adequate calories while adhering to the low fat regimen. The initial priority is to maintain weight. Once this is achieved, a patient may begin incorporating lower fat food choices if the low fat diet is still desirable. Diabetic diets have undergone significant changes since 1994. The emphasis is on individualizing the diet based on the medical nutrition needs.

Alterations in cognitive skills, ability to attend, or to speak will of course affect communication and social interaction and have implications for a patient's compliance with treatment objectives. Such changes may also trigger depression or a diminished sense of well being, with resultant decreased appetite and failure to maintain weight.

Pediatric feeding assessment: Pediatric feeding problems have been recognized in the literature for the past 30 years. Although no comprehensive definition of pediatric feeding problems has been widely accepted, infants and children 
experiencing feeding difficulties demonstrate either a refusal to eat orally and / or are unable to sustain oral feedings to maintain adequate caloric intake. Numerous articles have identified groups of children who are at risk for feeding problems. See table for etiologies commonly associated with feeding problems.

\section{Common etiologies associated with feeding problems}

a. Neurological dysfunction.

b. Gastrointestinal disease/dysfunction.

c. Cardio-respiratory compromise.

d. Sensory deprivation.

e. Structural anomalies.

f. Social-behavioral maladaptation.

A feeding assessment is a comprehensive, systematic biopsychosocial approach to the evaluation of a child who has feeding difficulties. The assessment can help professionals identify potential or actual feeding problems and develop appropriate interventions.

\section{Pediatric Feeding Assessment}

a. The feeding assessment consists of five parts:

b. Case history (obtained prior to evaluating child)

c. Nutritional screening, Feeding history, Developmental milestones (obtained from parent or caregiver's interview)

d. Physical Assessment

e. Oral reflexes and feeding skills assessment

f. Psychosocial international assessment.

\section{Case History}

Done before the evaluation of the child. It includes a review of the infant or child's:
a. Medical history.
b. Growth chart.
c. Current clinical nutritional status.

\section{Medical history}

The medical history review includes evaluating the perinatal and neonatal history, medical diagnosis, previous hospitalizations, and significant illnesses. The perinatal and neonatal history may provide information detailing any fetal distress, the infant's response during delivery, prematurity, any significant congenital anomalies, and any major illness during the first month of life. Congenital anomalies, central nervous system insults, or chronic illnesses may affect the child's ability to eat orally. Previous hospitalizations and significant illness can influence the child's developmental skills including feeding skills. Child's refusal to eat, oral motor organization, and / or ability to sustain adequate oral intake.

Growth chart: It is critical to review to growth chart to determine the child's nutritional status. Prolonged inadequate caloric intake results in an infant or child nutritionally failing to thrive. A child is defined as having failure to thrive when:

a. The weight for length ratio is $<5 \%$

b. The weight is $<5 \%$ or

c. The weight percentage has decreased two standard deviations or more.

Other growth charts for some specific patient populations, such as Down's syndrome and premature infants, are available.

Current clinical nutritional status: A review of the current clinical nutritional status should include laboratory tests and anthropometrics. Laboratory tests can help define nutritional deficiencies. The most readily available screening tests are the complete blood count and a chemistry panel. Protein deficiency (i.e., a low albumin and total protein). Anthropometric assessment measures triceps, skin fold thickness and mid arm circumference as a serial indicator of body fat and muscle mass. Anthropometrics may be a preferred measurement of nutritional status compared to growth charts when the infant or child's age is unclear.

Nutritional screening, feeding history and development milestones: This information should be elicited from the parent or caregivers. The feeding history needs to include the parent's perception of the feeding problem or difficulty and a thorough description of the child's mealtimes. The history should include type of food, amounts, textures, duration of feeding, physical environment, and family members usually present, the child's behavior, and any interventions tried. A 24-hour dietary recall of the child's feeding routine is helpful to assess individual nutritional patterns.

\section{Physical assessment}

An assessment of the child's general physical appearance and findings will provide information about the child's nutritional status. When performing this assessment, one must consider the infant or children;
a. Behavior.
b. Development.
c. Physical appearance.

Behavior: When observing the behavioral state, observe how the child acts. Is the child alert, active, irritable or apathetic? The alert and rested child provides the most realistic information about feeding behaviors. Irritability and apathy are commonly seen with malnutrition.

Development: The developmental assessment involves observing the child's fine and gross motor skills and muscle tone. Is the child performing tasks at the expected age, or is the child showing some developmental delays? Most children who are developmentally delayed exhibit some alteration in muscle tone, either hypertonia or hypotonia. Both the child's motor skills and muscle tone influence his or her ability to eat. 
Physical appearance: The physical appearance involves assessing the child's:
a. Skin.
b. Hair.
c. Eyes.
d. Mouth and oral cavity.

Skin: First, check the skin for color, bruises, rashes, and turgor. A pale color may indicate iron-deficiency anemia. Bruising may be due to vitamin $\mathrm{K}$ deficiency. Essential fatty acids, zinc or vitamin deficiencies are known to cause skin rashes. When inadequate fluid intake accompanies poor caloric intake, the skin will be dry. Loose skin covering the decreased subcutaneous fat indicates both a calorie and protein inadequacy (marasmus). Excessive fluid retention resulting in edema may be due to insufficient protein intake (kwashiorkor) or electrolyte imbalances.

Hair: Check hair for texture, color and distribution. Hair that is brittle, pale blond colored and sparsely distributed is seen with protein malnutrition.

Eyes: Check eyes for hydration status and infection. Xerophthalmia, or dryness, may be due to vitamin A deficiency. Malnutrition can affect the immune system and cause conjunctivitis.

Mouthand oral cavity: The physical appearance of the mouth and oral cavity portion are checked as part of the oral reflexes and feeding skills evaluation.

Psychosocial feeding assessment: The relationship and interaction between parents and infant or caregiver are important. This interaction may have profound effects on the child's nutrition and feeding. For ex, the parents may be confused and not offer food to their infant if the infant does not provide clear hunger cues (ie, agitation, crying and mouth opening). In cobtrast, the infant who does provide clear cues may not be offered food coz parents are insensitive to those cues. Therefore the quality of interaction between parents and their infants have a very significant impact on the amount of food ingested even if the infant has normal feeding skills. An interaction assessment tool such as Nursing Child Assessment Feeding Scale (NCFAS).
This provides information about the relationship between the child and parents.

Oral reflexes and feeding skills: The infant or child's reflexes and feeding skills determine the types of foods safely handled. During the first 3 years of life, dramatic oral motor and developmental feeding skill changes have profound effects on the types of food, textures, and feeding methods and the infant/child can safely control. The approach recommended for assessing oral reflexes and feeding skills is to progress from the least frightening or threatening (external touching of the face and mouth) to the most threatening (internal inspection of the mouth).

Some oral reflexes are common to all ages, but the most rapid oral reflex are feeding skills changes occur in infancy. Therefore, the oral reflexes and feeding skills assessments will be divided into eight infant developmental stages covering the 3 years of life.

\section{References}

1. Logemann JA (1984) Evaluation \& treatment of swallowing disorders.

2. Leonard R, Kendall $\mathrm{K}$ (2008) Dysphagia assessment and treatment planning: A team approach. ( $3^{\text {rd }}$ edn), plural publishing, USA, p. 384.

3. Hegde MN, Provencio-Arambula M, Provencio D (2007) Assessment of dysphagia in adults.

1. Murdoch BE, Cichero JAY (2006) Dysphagia foundation, theory and practice. John Wiley \& Sons, Ltd., New York, USA, pp. 1-582.Surg 52(1): 120-123.

2. Popovtzer A, Shpitzer T, Bahar G, Feinmesser R, Segal K (2006) Thyroid cancer in children: management and outcome experience of a referral center. Otolaryngol Head Neck Surg 135(4): 581-584.

3. Jarzab B, Handkiewicz Junak D, Włoch J, Kalemba B, Roskosz J, et al (2000) Multivariate analysis of prognostic factors for differentiated thyroid carcinoma in children. Eur J Nucl Med 27(7): 833-841.

4. Borson CF, Causeret S, Lifante JC, Augros M, Berger N, et al. (2004) Predictive factors for recurrence from a series of 74 children and adolescents with differentiated thyroid cancer. World J Surg 28(11): 1088-1092.

5. Lloyd RV, Buehler D, Khanafshar E (2011) Papillary thyroid carcinoma variants. Head Neck Pathol 5(1): 51-56.

6. Massimino M, Podda M, Spinelli C,Bleyer A (2017)ThyroidCancer in Cancer in Adolescents and Young Adults, PediatricOncology, A. Bleyer, et al. Springer International Publishing

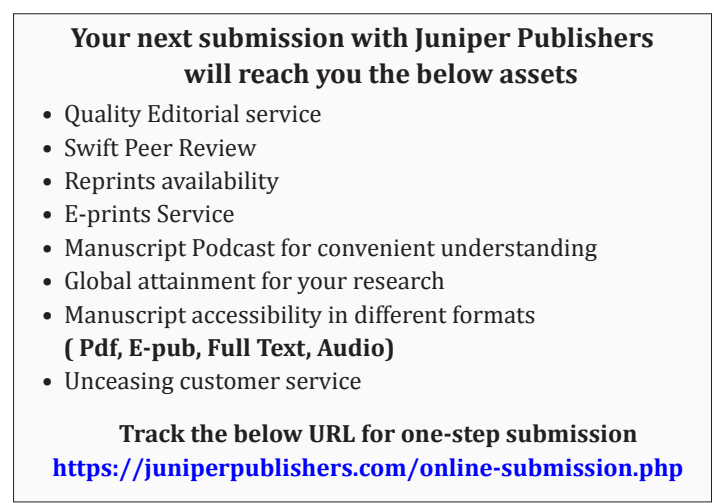

